warfarin therapy. Between 1961 and 1967 we started our first trial of oral anticoagulants in patients with advanced cancers and 29 patients out of 96 responded for one to five years. ${ }^{4}$

In 1967-8 it was not possible to organize a controlled clinical trial because of the small number of suitable patients available to us and the multiplicity of factors in each case. As an alternative, however, we used patients as their own controls by starting warfarin therapy when their disease became resistan to cytotoxic therapy. The results were presented at the annual meeting of the Irish Association for Cancer Research in December 1971 and are being published. Briefly, 30 cases of lymphoma and chronic leukaemia being maintained on a single cytotoxic agent -busulphan, chlorambucil, or cyclophosphamide-were given warfarin therapy to double the prothrombin time when their disease became resistant. The same cytotoxic agent was continued in each case Response was measured by calculating the total dosage of cytotoxic agent, per three month periods, required to maintain the patient in remission before and after the addition of warfarin. Twenty-one cases responded and the three-monthly average requirement of cytotoxic agent for these patients decreased to $25 \%$ of the prewarfarin level after three months.

From our experience with warfarin in the treatment of human cancer it would appear to be a useful agent for maintenance therapy This supports the observation of Michaels, quoted in your leading article, that mortality from cancer is lowered by oral anticoagulants We found that morbidity is also lowered and this is usually the first sign of response to therapy occurring seven to 14 days after the prothrombin time is doubled.-I am, etc.,

R. Douglas Thornes Department of Experimental Medicine, Roval College of Surgeons in Ireland,

Dublin 2

1 Thornes, R. D., and Martin, W. T., Irish fourma of Medical Science, 1961, 6, 487.

2 Lisnell, A., and Mellgren, J., Acta Pathologica Microbiologica Scandinavica, 1963, 57, 145.

Thornes, R. D., Edlow, D. W., and Wood, S. inr., The 4 Thornes, R. D., Fournal of the Irish Medical
Association, 1969, 62, 426.

** The reference to A. S. Ketcham et al., should have read: American fournal of Roentgenology, 1971, 3, 42.-ED., B.M.f.

\section{Hospital Advisory Service}

SIR,-When the Hospital Advisory Service's courteous teams visited one mental hospital in 1971 I found much of their advice instructive, stimulating, and helpful. I have also had the pleasure of reading Dr. A. A Baker's Annual Report for $1970 .^{1}$ Nevertheless, I strongly endorse Dr. J. L. Crammer's plea (18 December, p. 746) that they ought to improve their methods of analysing work loads and the staff needed to labour under them; they should publish their standards, and key statistics of the hospitals they visit. Inadequate staff ratios may delay the closure of mental hospitals, but the greater risk is that the standard of care afforded existing inpatients will fall below an acceptable level

Wing and his colleagues ${ }^{2}$ have already established in three mental hospitals that the progress of earlier years has begun to slip back, and they emphasize how difficult it is to sustain social innovations. One explanation is that because of the present steadily increasing work load "the attention that doctors can give to long-stay patients, and the number of staff who can be spared for rehabilitation, must inevitably diminish."

A logical strategy, where general hospital units have yet to open, is to arrange the existing mental hospital (or each catchment division within it) in two sections: admission wards and other wards. Each admission unit would look after the total inpatient load of its catchment area (and in doing so would not bequeath patients to the other wards) until the general hospital department opens.

To do this staff ratios similar to those of the general hospital units are essential. A common ratio for junior medical staff is three doctors (senior house officers or registrars) for 60 beds serving a population of 120,000 . For the remainder of the mental hospital it would be inequitable if 100 patients had fewer than one junior doctor to look after them, particularly when the aim is to rehabilitate and support in the community as many as feasible. The numbers of other staff also need careful and local evaluation. For example the nursing load provided by incontinent severely demented patients, a proportion of whom are cared for in mental hospitals, is extremely high. ${ }^{3}$

If I understand the role of the Hospital Advisory Service correctly one of their main tasks is to consider staff ratios within the hospitals visited. Yet there is a notable lack of facts about these in the Annual Report for 1970. The mental hospital already mentioned has 18 junior doctors (below senior registrar grade), but on the unexceptional staff ratios outlined it needs about 30 Yet the Hospital Advisory Service considered that this hospital was relatively well provided with non-consultant psychiatric staff. The Advisory Service's standard was not revealed. Should it be revised?-I am, etc.

London N.W.3

H. G. S. SERGEANT

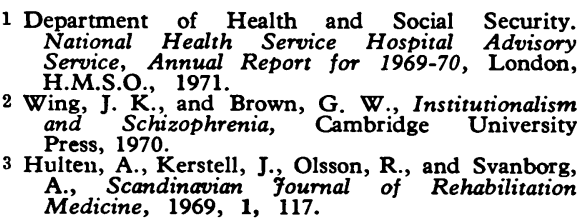
Medicine, 1969, 1, 117.

\section{Immune Suppression, Gliomas, and} Tubersulosis

SIR,-The possibility that some cancers may be due to defects in immunological surveillance has been widely discussed of late. The theory states that many thousands of cell divisions occur each day within the human body and a number of faults in the mitotic process are inevitable as the copying or template mechanism for DNA replication must break down from time to time. Some of these "mutants" may well be "potential tumours," and it is suggested that the immune system recognizes and destroys them. Thus several potential cancers may occur in an individual every day but these are usually completely suppressed. It is envisaged, therefore, that the immune system has two main functions-its classically recognized role as a defence mechanism against external attack by foreign organisms, mediated usually by humoral antibody, and the problem of internal security, perhaps effected by the cellular immune system. Within the complex ecology of the body involving massive cell populations the latter role of internal security may well be the more important.

Evidence for this hypothesis has been admirably documented and discussed by Burnet in his book on Immunological Surveillance. Two of the most important pieces of evidence are, firstly, that there is a high risk of malignancy, particularly intracerebral lymphomas, after immunosuppression in transplant recipients, and, secondly, that neoplasms are a common complication in certain immune deficiency states of genetic origin. It therefore occurred to us that it might be worthwhile looking into the medical histories of patients with cerebral gliomas to see whether there was an increased incidence of conditions likely to be associated with a deficiency in the immune system.

We accordingly determined the previous medical history of 26 patients with cerebral gliomas, and as a control series took 52 patients with other conditions. The positive finding related to the incidence of old tuberculosis as defined by the history and/or radiological changes. There were six cases of old tuberculosis among the $\mathbf{2 6}$ gliomas compared with 5 cases among the 52 controls. The difference is not statistically significant, but the finding that nearly a quarter of the patients with gliomas had a previous history of tuberculosis is certainly impressive. Also of interest is the fact that among the 26 gliomas there were two cases of diabetes (one also had tuberculosis), one of hepatic cirrhosis, and one of long-standing chronic chest infection-this last in a female. Thus a third of the patients with gliomas had a previous medical history containing a major disease which could conceivably be associated with some alteration in immune response.

This is a small and preliminary study and the results could well be due to chance, but, if confirmed in larger series, the results would carry some very interesting implications. They would provide further evidence that infection with tuberculosis is in large part governed by the resistance of the host, and that many patients with this disease may have occult immune deficiencies. They would also provide evidence in support of the theory that neoplasia is, at least in part, due to a deficiency in immunological surveillance.-We are, etc.,

Ronald Finn D. W. WARD

Royal Southern Hospital, M. L. MATtisoN

Royerpool

1 Lancet, 1971, 2, 143. Pergamon Press, 1970.

\section{International Convention on Psychotropic} Drugs

SIR,- The new international convention on psychotropic substances adopted at a United Nations conference in Vienna in $1971^{1}$ raises some fundamental questions about the control of scientific research-particularly research that requires possession of controlled drugs for research purposes. Some of the clauses of the new convention are open to a wide range of administrative and legal interpretation.

One clause in the preamble reads: "Recognizing that the use of psychotropic 